\title{
Acid-Sensing Ion Channels in Postoperative Pain
}

\author{
Emmanuel Deval, ${ }^{1}$ Jacques Noël, ${ }^{1}$ Xavier Gasull ${ }^{1,2}$ Anne Delaunay, ${ }^{1}$ Abdelkrim Alloui, ${ }^{3,4}$ Valérie Friend, ${ }^{1}$ \\ Alain Eschalier, ${ }^{3,4}$ Michel Lazdunski, ${ }^{1}$ and Eric Lingueglia ${ }^{1}$ \\ ${ }^{1}$ Institut de Pharmacologie Moléculaire et Cellulaire, Unité Mixte de Recherche 6097 Centre National de la Recherche Scientifique/Université de Nice-Sophia \\ Antipolis, 06560 Valbonne, France, ${ }^{2}$ Laboratory Neurophysiology, Facultat de Medicina, Universitat de Barcelona-Institut d'Investigacions Biomèdiques \\ August Pi i Sunyer, 08036 Barcelona, Spain, ${ }^{3}$ Inserm, U 766, F-63001 Clermont-Ferrand, France, and ${ }^{4}$ Clermont Université, Université d'Auvergne, \\ Pharmacologie fondamentale et clinique de la douleur, BP 10448, F-63000 Clermont-Ferrand, France
}

Iatrogenic pain consecutive to a large number of surgical procedures has become a growing health concern. The etiology and pathophysiology of postoperative pain are still poorly understood, but hydrogen ions appear to be important in this process. We have investigated the role of peripheral acid-sensing ion channels (ASICs), which form depolarizing channels activated by extracellular protons, in a rat model of postoperative pain (i.e., hindpaw skin/muscle incision). We report high levels of ASIC-type currents $(\sim 77 \%)$ in sensory neurons innervating the hindpaw muscles, with a prevalence of ASIC3-like currents. The ASIC3 protein is largely expressed in lumbar DRG neurons innervating the plantar muscle, and its mRNA and protein levels are increased by plantar incision $24 \mathrm{~h}$ after surgery. Pharmacological inhibition of ASIC3 channels with the specific toxin APETx2 or in vivo knockdown of ASIC3 subunit by small interfering RNA led to a significant reduction of postoperative spontaneous, thermal, and postural pain behaviors (spontaneous flinching, heat hyperalgesia, and weight bearing). ASIC3 appears to have an important role in deep tissue but also affects prolonged pain evoked by skin incision alone. The specific homomeric ASIC1a blocker PcTx1 has no effect on spontaneous flinching, when applied peripherally. Together, these data demonstrate a significant role for peripheral ASIC3-containing channels in postoperative pain.

\section{Introduction}

Pain caused by surgical procedures is a significant clinical problem. Persistent postoperative pain occurs in patients undergoing thoracotomy, coronary artery bypass surgery, inguinal hernia repair, cesarean section, limb amputations, and breast, gallbladder, or lung surgeries (for review, see Perkins and Kehlet, 2000; Bove et al., 2009). Patients report both resting and evoked pain, the latter being caused by coughing, movement, or pressure application on or near the wound (Moiniche et al., 1997). Intensity of acute postoperative pain is a predictor of chronic pain (Perkins and Kehlet, 2000) and often complicates the recovery process after surgery (Pavlin et al., 2002), prolonging hospitalization, predisposing patients to greater morbidity, and increasing stress (Wu et al., 2003; Duarte et al., 2005). Optimizing pain management after surgery is thus important to enhance patient comfort and expedite recovery.

The etiology of postoperative pain is still poorly understood, and the mechanisms involved are probably multiple, including tissue injury related to the incision itself, secondary inflammation, and damage to nerves caused by tissue retraction during the

Received Oct. 4, 2010; revised Feb. 7, 2011; accepted Feb. $26,2011$.

Author contributions: E.D., J.N., and E.L. designed research; E.D., J.N., X.G., A.D., A.A., V.F., and E.L. performed research; E.D., X.G., A.D., and E.L. analyzed data; E.D., A.E., M.L., and E.L. wrote the paper.

We thank Drs. Anne Baron, Sylvie Diochot, and Miguel Salinas for helpful discussion, and C. Chevance for secretarial assistance. We thank the Association Française contre les Myopathies, the Agence Nationale de la Recherche, the Institut UPSA de la Douleur, the Association pour la Recherche sur le Cancer, the Fondation pour la Recherche Médicale, and Grant FIS08/0014 (from Institut Salud Carlos III, Spain) for financial support.

The authors declare no conflict of interest.

Correspondence should be addressed to Emmanuel Deval at the above address. E-mail: deval@ipmc.cnrs.fr.

DOI:10.1523/JNEUROSCI.5266-10.2011

Copyright $\odot 2011$ the authors $\quad 0270-6474 / 11 / 316059-08 \$ 15.00 / 0$ surgery (Flatters, 2008). The mechanisms of postincisional nociception are distinct from other pain conditions (Pogatzki-Zahn et al., 2007). Hydrogen ions appear as key mediators in this process, and local decrease of tissue $\mathrm{pH}$ is correlated with pain behaviors in rat models of surgical pain stemming from skin and/or muscle incision (Woo et al., 2004). The transient receptor potential vanilloid 1 (TRPV1) channel has been reported to contribute to heat hypersensitivity after plantar incision in mice (Banik and Brennan, 2009). On the other hand, nonselective or nonsubtype-specific inhibitors of acid-sensing ion channels (ASICs), such as amiloride and A-317567, have also suggested a possible implication of these channels in postoperative pain (Dubé et al., 2005). ASICs form a family of voltage-independent cation channels activated by extracellular acidification and expressed by nociceptors (Waldmann et al., 1997a,b; Chen et al., 1998; Bässler et al., 2001).

In the present work, we have investigated the role of peripheral ASICs in rat postoperative pain developed following hindpaw skin/muscle incision (Brennan et al., 1996). We report high levels of ASIC3-type currents and a large expression of the protein in lumbar DRG neurons innervating the rat hindpaw muscles. Plantar incision is associated with an increase in ASIC3 mRNA level and protein expression in lumbar sensory neurons. The pharmacological blockade of ASIC3 with the specific sea anemone toxin APETx2 (Diochot et al., 2004) and the in vivo knockdown of ASIC3 expression with a specific small interfering RNA (siRNA) (Deval et al., 2008) both led to a significant reduction of postoperative pain behaviors, supporting the implication of peripheral ASIC3 in postoperative pain. 


\section{Materials and Methods}

Primary culture of labeled dorsal root ganglia neurons. Lumbar (L3-L6) DRGs were dissected bilaterally from adult male Wistar rats previously injected intramuscularly with the retrograde fluorescent dye DiI $(5 \times 1$ $\mu \mathrm{l}$ into hindpaw muscles). After an enzymatic dissociation with $0.1 \%$ collagenase, neurons were then plated on collagen-coated $35 \mathrm{~mm}$ Petri dishes (Biocoat) and maintained in culture at $37^{\circ} \mathrm{C}\left(95 \%\right.$ air $\left./ 5 \% \mathrm{CO}_{2}\right)$ in DMEM containing $5 \%$ fetal calf serum. Electrophysiological experiments were performed $1-8 \mathrm{~d}$ after plating. Culture media were changed $1 \mathrm{~d}$ after plating and then every $3 \mathrm{~d}$ of culture by replacing half the media. ASIC currents were only considered when their amplitude was larger than $30 \mathrm{pA}$. The culture conditions did not dramatically change the properties of ASIC-like currents. A total of $33.3 \%$ of the cells (7 of 21 neurons) have been used 1-2 d after plating, and 66.7\% (14 of 21 neurons) have been used $>2 \mathrm{~d}$ after plating (between 3 and $8 \mathrm{~d}$ ). A total of $71.4 \%$ of $1-2$-d-old neurons ( 5 of 7 neurons) and $78.6 \%$ of older neurons (11 of 14 neurons) presented $\mathrm{pH}$ 6.6-evoked ASIC-like current, with 20 and $18.2 \%$ of type 1 current, and 80 and $72.7 \%$ of type 2 current, respectively. One neuron in the older population expressed an ASIC-type current partly inhibited by the PcTxl toxin and with an intermediate inactivation rate that was not classified as type 1 or type 2 .

Electrophysiology. We used the whole-cell configuration of the patchclamp technique to measure membrane currents (voltage-clamp) or membrane potentials (current-clamp). Recordings were made at room temperature using an RK-400 amplifier (Bio-Logic Science Instruments) with a $3 \mathrm{kHz}$ low-pass filter (Krohn-Hite). Data were sampled at $10 \mathrm{kHz}$, digitized by a Digidata 1322A A-D/D-A converter (Molecular Devices), and recorded on a hard disk using pClamp software (version 9; Molecular Devices). Patch pipettes (1-4 M $\Omega$ ) contained the following (in $\mathrm{mM}$ ): $135 \mathrm{KCl}, 2.5 \mathrm{Na}_{2}$-ATP, $2 \mathrm{MgCl}_{2}, 2.1 \mathrm{CaCl}_{2}, 5$ EGTA, 10 HEPES, pH 7.25 with $\mathrm{KOH}$. Various pH-buffered solutions and drugs of interest were applied to individual patch-clamped cells using a homemade microperfusion system driven by microsolenoid valves (Sirai) allowing rapid solution changes. The control bath solution contained the following (in mM): $145 \mathrm{NaCl}, 5 \mathrm{KCl}, 2 \mathrm{MgCl}_{2}, 2 \mathrm{CaCl}_{2}, 10$ HEPES, pH 7.4 with $\mathrm{NaOH}$. ASIC currents were induced by shifting one of eight outlets of the microperfusion system from the $\mathrm{pH} 7.4$ control solution to an acidic test solution. For the experiments performed on cultured DRG neurons, glucose $(10 \mathrm{~mm})$ was added to the control bath solution.

Quantitative RT-PCR experiments. Changes in rat ASIC3 expression in the postoperative pain model were evaluated in lumbar dorsal root ganglia by reverse transcription quantitative PCR (RT-qPCR) $24 \mathrm{~h}$ after surgery. L4-L6 ganglia from both sides of the animal were removed, RNA was extracted with the RNeasy micro kit (Qiagen), and cDNA was prepared with cloned AMV First-Strand cDNA synthesis kit (Invitrogen) and used for qPCR in a Light-Cycler480 (Roche Products). ASIC3 expression was compared between ipsilateral and contralateral sides for the postoperative pain model and for naive animals that did not have any surgery. Primers used are as follows: $18 \mathrm{~S}$ aagtccetgccetttgtacaca/gatccgagggcctcactaaac; and ASIC3 cacccaatgacttgcactgg/taggcagcatgttcagcagg. For each animal, ASIC3 expression was normalized against $18 \mathrm{~S}$ and converted to fold induction relative to the contralateral side.

Retrograde labeling of hindpaw muscle afferents and immunohistochemistry. Dorsal root ganglion neurons innervating the plantar hindpaw muscle in male Wistar rats ( $8-11$ weeks) were labeled by intramuscular injections of $3 \times 2 \mu \mathrm{l}$ of the fluorescent dye fast blue (Polysciences) at a concentration of $2 \%$ in saline. Dye was injected 2 weeks before killing the rats, and lumbar (L5) DRGs were removed and processed for immunohistochemistry. Freshly isolated DRGs were embedded in Tissue-Tek compound (Sakura Finetek, Bayer Diagnostic) and rapidly frozen in isopentane cooled by liquid nitrogen. Cryostat sections (12 $\mu \mathrm{m}$ thickness) were then prepared. Sections were fixed in $4 \%$ paraformaldehyde, permeabilized with $0.1 \%$ Tween 20 , and blocked with $2 \%$ horse serum before overnight incubation at $4^{\circ} \mathrm{C}$ with an anti-ASIC3 rabbit polyclonal antibody (1:400; Alomone Labs) followed by a goat anti-rabbit Alexa 594 secondary antibody (1:200; Invitrogen). Specificity of the primary antibody was previously confirmed on ASIC3 knock-out mice (Ikeuchi et al., 2009) and on the recombinant channel (data not shown). Staining was visualized using an Axiovert 200M microscope (Carl Zeiss). Fast bluelabeled neurons were identified by their blue fluorescence following exposure to ultraviolet light at $350 \mathrm{~nm}$. The images were analyzed with ImageJ software (National Institutes of Health). ASIC3 expression was quantified as the percentage of total fast blue-positive neurons. Consecutive slices were not used to avoid double counting.

Animal handling and plantar incisional pain model. Adult male Wistar rats (Charles River) were housed with ad libitum access to food and water, in a temperature-controlled room with a $12 \mathrm{~h} \mathrm{light/dark} \mathrm{cycle,} \mathrm{at}$ least 1 week before the surgical procedure. The plantar incisional pain model was then operated as initially described by Brennan et al. (1996). Rats were anesthetized with isoflurane $2.5 \%$, and the skin of the operated foot was sterilized with povidone iodine 10\% (Merck) and placed in the hole of a sterile drape. A $1 \mathrm{~cm}$ longitudinal incision was made through skin and fascia of the plantar face of a hindpaw, starting at $0.5 \mathrm{~cm}$ from the proximal edge of the heel. The plantar muscle was then elevated with forceps and incised longitudinally with the blade keeping attention to leave muscle origins intact. After hemostasis, the wound was apposed with two sutures of 5/0 polyamide monofilament (Filapeau, Peters Surgical). Vehicle ( $20 \mu \mathrm{l}$ of $\mathrm{NaCl} 0.9 \%)$, APETx $2(20$ $\mu \mathrm{l}$ at $20 \mu \mathrm{M})$, or PcTx1 $(20 \mu \mathrm{l}$ at $120 \mathrm{nM})$ was applied directly to the wounds using a micropipette just before the second suture was made. The concentrations of APETx 2 and PCTx 1 used correspond to six times the concentrations that produce the maximal inhibitory effects on recombinant ASIC3 and ASIC1a currents, respectively. For recovery from anesthesia, animals were placed in new cages with special nonabrasive dry litter (Litaspen Premium, SPPS). Behavioral pain tests were performed 4 and $24 \mathrm{~h}$ after surgery.

Intrathecal injection of siRNA. Ten microliters of a siRNA $(2 \mu \mathrm{g}) / \mathrm{i}-\mathrm{Fect}$ (Neuromics) mix was injected intrathecally between the L4 and L5 vertebrae of rats using a Hamilton syringe and a 25 gauge needle. Animals received one injection per day for $4 \mathrm{~d}$ (Fig. $4 \mathrm{~A}$, protocol). ASIC3 (CUACACGCUAUGCCAAGGAdtdt) and the corresponding scramble (GCUCACACUACGCAGAGAUdtdt) siRNAs have been previously described (Deval et al., 2008).

Behavioral pain tests. Spontaneous postoperative pain was assessed by counting the number of spontaneous flinches of the operated paw (flinching test). Rats were placed in transparent plastic boxes, and the number of flinches was counted over a period of $5 \mathrm{~min}$.

Thermal hyperalgesia was measured with the radiant heat test (Bioseb). Rats were placed in transparent plastic boxes on an elevated floor. A radiant heat source was then placed under one animal foot and maintained until the rat lifted its foot.

A dynamic weight-bearing test (Bioseb) measures the postural equilibrium on freely moving rats. The rats were placed in a transparent chamber $(30 \times 30 \mathrm{~cm})$ on a calibrated weight transducer pad. Animals were moving freely in the chamber, and the transducer measured the repartition of the rat's average body weight on each paw over a 5 min time period. Naive rats equilibrate equally their body weight on each hindpaw, and a difference of weight distribution reflects pain experienced by rats. The weight-bearing difference was calculated as the absolute value of the difference between the weights on the two hindpaws.

Statistical analysis. Data are presented as the mean \pm SEM. Statistical differences between different sets of data were assessed by performing parametric or nonparametric tests where appropriate (see figure legends).

\section{Results}

ASIC-type currents activated by moderate acidifications in sensory neurons innervating the hindpaw muscles of the rat ASIC channel functional properties were evaluated in rat DRG neurons innervating the hindpaw muscles identified by retrograde labeling with the fluorescent dye DiI. Whole-cell voltageclamp currents evoked by extracellular acidification from a resting $\mathrm{pH} 7.4$ to a test $\mathrm{pH} 6.6$ were recorded. The value for the test $\mathrm{pH} 6.6$ was chosen because it corresponds to the tissue $\mathrm{pH}$ measured in vivo in rat muscle incision models of postoperative pain (Woo et al., 2004). We found that $77 \pm 13 \%$ of the sensory 
A

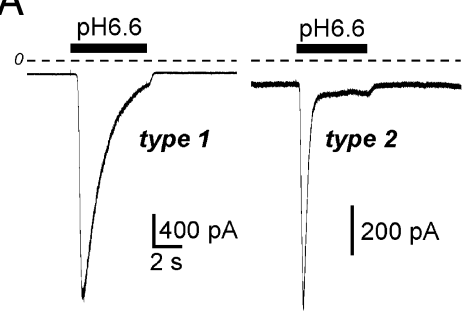

C

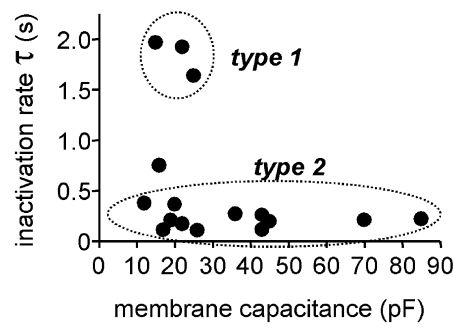

E

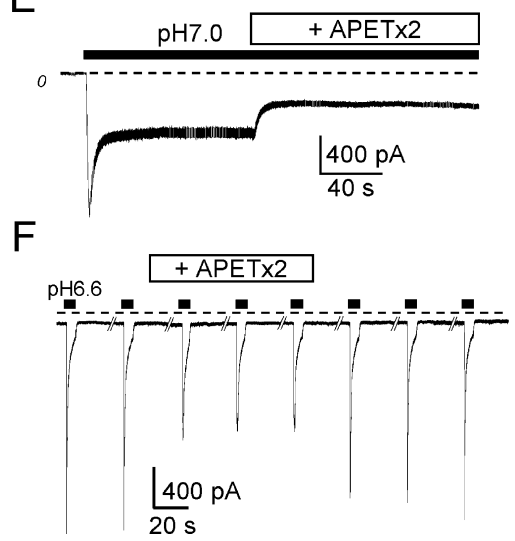

B

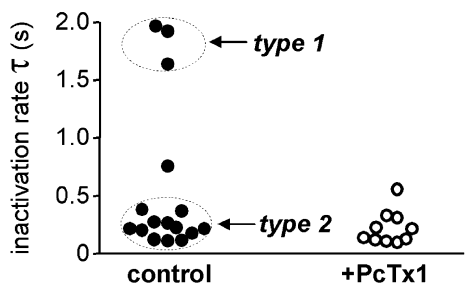

D

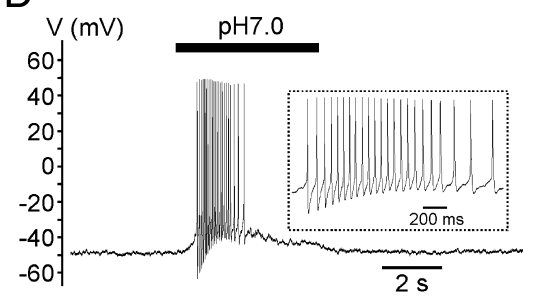

G
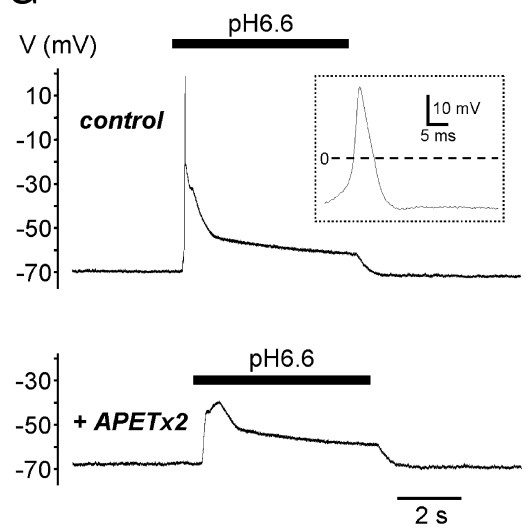

Figure 1. Electrophysiological characterization of ASIC channels in DRG neurons innervating the rat hindpaw muscles. $\boldsymbol{A}$, Typical ASIC currents recorded in voltage-clamp (holding potential $-80 \mathrm{mV}$ ) from retrogradely labeled sensory neurons innervating hindpaw muscles. Current was activated by a rapid switch of the external pH from 7.4 to 6.6 , as indicated by the black bars above the current traces. The dashed line represents the zero current level. According to their inactivation rates $(\tau)$, two main types of ASIC current can be distinguished (type 1 and type 2). $\boldsymbol{B}$, Analysis of the inactivation rate of native pH 6.6-induced ASIC currents in control condition and in the presence of the PcTx1 toxin. The slow-inactivating type 1 current is blocked by the toxin. C, Functional distribution of type 1 and type 2 ASIC currents as a function of the neuron membrane capacitance. D, Current-clamp experiment performed on a neuron exhibiting a type 2 ASIC current. A slight acidification of the external medium to pH 7.0 depolarizes the membrane sufficiently to trigger firing. Action potentials are magnified in the inset. $\boldsymbol{E}$, Effect of APETx2 on recombinant sustained ASIC3 current recorded at $-80 \mathrm{mV}$ from F11-transfected cells. Current was activated by external $\mathrm{pH}$ changes from $\mathrm{pH} 7.4$ to 7.0, as indicated by the black bar. The APETx2 toxin was applied externally at $1 \mu \mathrm{m}$, as indicated. $\boldsymbol{F}$, Effect of APETx2 (5 $\mu \mathrm{m})$ on native pH 6.6-evoked ASIC current recorded at $-80 \mathrm{mV}$ from retrogradely labeled sensory neurons innervating hindpaw muscles. The fast component of the current (type 2) is largely inhibited by the toxin. G, Current-clamp experiment performed on the same neuron as in $\boldsymbol{F}$. The depolarization induced by the $\mathrm{pH}$ 6.6-evoked ASIC current was sufficient to trigger an action potential (magnified in the inset). The presence of APETx2 inhibits the pH 6.6-evoked depolarization and the action potential threshold is not reached.

neurons innervating the hindpaw muscles presented transient current with ASIC-like kinetics when exposed to $\mathrm{pH} 6.6$ (data from 2 different cultures, $n=9$ of 10 and 7 of 11 neurons, respectively). The ASIC-expressing neurons had an average membrane capacitance of $32.3 \pm 5.2 \mathrm{pF}$ and an average resting potential of $-53.4 \pm 2.9 \mathrm{mV}$. Two different types of ASIC currents were observed and were readily distinguished based on their inactivation kinetics (Fig. 1A). Type 1 current exhibited a slow inactivating rate $(\tau=1841 \pm 104 \mathrm{~ms}$, present in 3 of 16 ASIC-expressing neurons) (Fig. $1 B$ ) similar to the inactivation rate observed for the current elicited by recombinant ASICla $(\tau=1712 \pm 93 \mathrm{~ms}$, $n=10$, data not shown) (Baron et al., 2008). This current was largely inhibited by the toxin PcTx1 (78 \pm $20 \%$ of inhibition of the current amplitude) (Fig. 1B), a specific blocker of ASIC1a homomeric channels (Escoubas et al., 2000). These data indicate that, in DRG neurons innervating hindpaw muscles, the slow-inactivating type 1 current was mainly carried by homomeric ASICla channels. Conversely, type 2 current had rapid inactivation kinetics $(\tau=217 \pm 26$ ms, present in 12 of 16 ASIC-expressing neurons) (Fig. $1 \mathrm{~B}$ ) close to the inactivation rates recorded for recombinant ASIC3 channels $(\tau=234 \pm 11 \mathrm{~ms}, n=14$, data not shown) (Waldmann et al., 1997b). PcTx1 had a small effect (32 $\pm 5 \%$ inhibition) on $70 \%$ of type 2 currentexpressing neurons (7 of 10 neurons tested), showing the presence of low levels of homomeric ASICla channels together with ASIC3-type current in these neurons. The type 2 current was inhibited by the toxin APETx2, which blocks both the peak and the window current of ASIC3containing channels (Diochot et al., 2004) (Fig. $1 E$ ). Indeed, application of APETx2 $(5 \mu \mathrm{M})$ on a neuron displaying a mixture of ASIC currents resulted in a 50\% inhibition of the peak amplitude evoked at $\mathrm{pH}$ 6.6 (Fig. $1 F$ ). Importantly, APETx2 also decreased neuronal excitability in response to pH 6.6 (Fig. 1G).

Finally, in 1 of 16 neurons expressing ASIC-type currents, the current kinetic displayed an intermediate inactivation rate $(\tau=752 \mathrm{~ms})$ (Fig. $1 B$ ). This current was partly inhibited by the PcTxl toxin (46\% of inhibition), and the remaining current had an intermediate inactivation rate $(\tau=550 \mathrm{~ms})$, which probably reflects the activity of a complex mix of homomeric ASIC1a and other heteromeric ASIC channels.

A detailed analysis of the whole-cell membrane capacitance of ASIC-type current-expressing neurons (Fig. 1C) showed that the type 1 current, mainly supported by homomeric ASICla channels, was expressed in small DRG neurons, with a cell capacitance of $\sim 20 \mathrm{pF}$, while the type 2 currents, mainly supported by ASIC3 channels, were distributed in both small and large DRG neurons with cell capacitance ranging from 10 to 85 pF. Endogenous type 2 current (i.e., ASIC3-type) conferred to sensory neurons the capacity to electrically respond to very small acidifications near the physiological $\mathrm{pH}$ since activation of this current by a shift from resting $\mathrm{pH} 7.4$ to $\mathrm{pH} 7.0$ was sufficient to depolarize the membrane and promote action potential firing (Fig. $1 D$ ). Together, these data indicate that in neurons innervating hindpaw muscles, ASIC3-containing channels, alone or together with homomeric ASIC1a channels, support most of the ASIC current elicited by moderate external acidosis in a range that is relevant to incisional pain $(6.6<\mathrm{pH}<7.4)$. 
ASIC3 is largely expressed in sensory neurons innervating the hindpaw plantar muscle

Functional data show a high level of ASIC3-type currents in rat DRG neurons innervating the hindpaw skeletal muscles, and we have analyzed the expression of the corresponding protein by immunohistochemistry. This analysis was done on sensory neurons innervating the plantar hindpaw muscle [i.e., the muscle incised in the model of postoperative pain of Brennan et al., (1996) used in the present study]. We identified this population of sensory neurons in L5 DRG by retrograde labeling through injection of the fluorescent dye fast blue in the plantar muscle followed by immunohistochemical staining for ASIC3. In naive rats, a large number of sensory neurons that innervate the plantar muscle expressed ASIC3 (75 \pm $2 \% ; n=4$ rats; 217 fast blue-labeled neurons analyzed) (Fig. 2A,B). Moreover, $\sim 62 \%$ of the ASIC3-labeled neurons have diameters in the range of those recorded with patch-clamp (i.e., from 10 to $45 \mu \mathrm{m}$ ), which is consistent with the percentage of sensory neurons that innervate the hindpaw muscles and exhibit ASIC3-type currents ( $~ 57 \%, 12$ of 21 ) (Fig. 1). Analysis of the soma size of the total population of ASIC3-expressing neurons showed a large expression in small-diameter neurons and a significant expression in medium- to large-diameter neurons (Fig. 2C) (674 ASIC3-positive neurons analyzed), which is in good agreement with previous results (Voilley et al., 2001; Molliver et al., 2005). The distribution of ASIC3 in neurons innervating the hindpaw plantar muscle (i.e., ASIC3 and fast blue-positive neurons) was slightly different from that of ASIC3 in the whole population of neurons, in which it was detected in a greater proportion of medium- to large-diameter neurons (Fig. 2C) (118 fast blue- and ASIC3-positive neurons analyzed). This distribution pattern is consistent with the large range of cell membrane capacitance observed on ASIC3-type neurons with the patch-clamp technique (Fig. 1C).

\section{ASIC3 contributes to the detection of postincisional pain}

Because ASIC3 is largely expressed in sensory neurons innervating the hindpaw muscles (including the plantar muscle), where it constitutes the main ASIC-type current (Figs. 1,2), we have analyzed its contribution to postoperative muscular pain in the plantar postincisional pain model in rat (Brennan et al., 1996). Postincisional pain behaviors were first analyzed $4 \mathrm{~h}$ after surgery (Fig. 3A) by measuring spontaneous flinching (Fig. 3B), heat hyperalgesia (Hargreaves test) (Fig. 3C), and hindpaw weight bearing (dynamic weight-bearing test) (Fig. 3D). Plantar muscle incision caused significant hyperalgesia in all three behavioral tests. We then tested the effect of pharmacological inhibition of ASIC3 by the specific inhibitory peptide APETx2 (Diochot et al., 2004). APETx 2 was known to block the peak of the acidinduced current flowing through homomeric and heteromeric ASIC3 channels (Diochot et al., 2004). We show here that APETx2 also inhibits the ASIC3 sustained window current
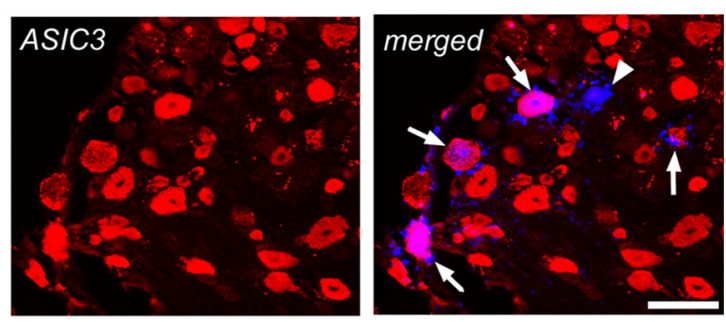

C

ASIC3 $\mathrm{ASIC} 3+\mathrm{FB}$

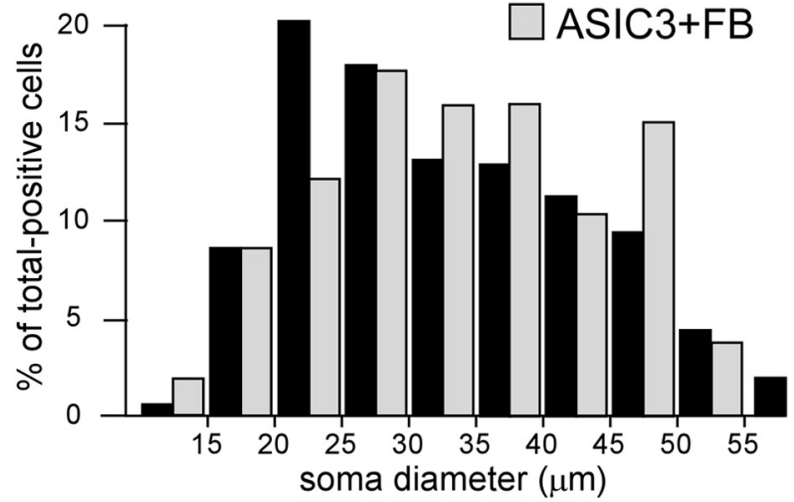

Figure 2. Analysis of ASIC3 expression in sensory neurons innervating the hindpaw plantar muscle. $A$, Immunolocalization of ASIC3 in lumbar DRG neurons. Fast blue-positive neurons retrogradely labeled from the plantar muscle (left, blue) and ASIC3or not expressing (arrowhead) ASIC3 (scale bar, $100 \mu \mathrm{m}$ ). $\boldsymbol{B}$, Percentage of fast blue (FB)-positive neurons expressing the ASIC3 ( 作 ASIC3 + FB-positive cells associated with different soma diameter ranges. The proportion of medium to large neurons expressing ASIC3 is larger in neurons that innervate the hindpaw muscle compared with the whole population of ASIC3-positive cells.

evoked by moderate external acidification to $\mathrm{pH} 7.0$ (Fig. $1 E$ ), an acidification well in the range of the tissue $\mathrm{pH}$ reached after surgical incision in the rat (Woo et al., 2004). This sustained current has been proposed to be important for pain perception (Yagi et al., 2006; Deval et al., 2008; Salinas et al., 2009). Local intraoperative application of APETx2 $(20 \mu \mathrm{l}, 20 \mu \mathrm{M})$ significantly reduced the spontaneous pain behavior of operated rats compared with vehicle-treated animals (Fig. 3B) (reduction of the numbers of flinches by $52 \%, p<0.001, n=19$ and 28 , respectively). Conversely, animals treated with PcTx1 (a potent and specific inhibitory spider toxin for homomeric ASIC1a channels; $20 \mu \mathrm{l}, 120$ nM) did not show any significant reduction in the number of flinches (Fig. 3B, left) $(n=10)$. The role of ASIC3 was further confirmed by in vivo knockdown of ASIC 3 expression through intrathecal injections of a specific siRNA targeting the ASIC3 mRNA (Fig. 3A). Uptake of this siRNA by lumbar DRG neurons innervating rat hindpaw and specific knockdown of ASIC3 mRNA versus other ASIC subunits and TRPV1 have been previously described (Deval et al., 2008). Rats treated with the ASIC3 siRNA displayed a significant decrease of the postoperative spontaneous pain behavior compared with animals injected with the control scramble siRNA (63\% reduction of the number of flinches, $p<0.05, n=10$ ) (Fig. $3 B$, right). The flinching score of rats treated with the scramble siRNA (Fig. 3B, right) was not significantly different from the score of vehicle-treated animals (Fig. $3 B$, left) ( $p=0.39$, unpaired $t$ test).

Hindpaw skin and muscle incisions have been shown to evoke primary heat hyperalgesia (Brennan et al., 1996) (i.e., hyperalgesia inside the area injured by the incision). Consistent with this 
A

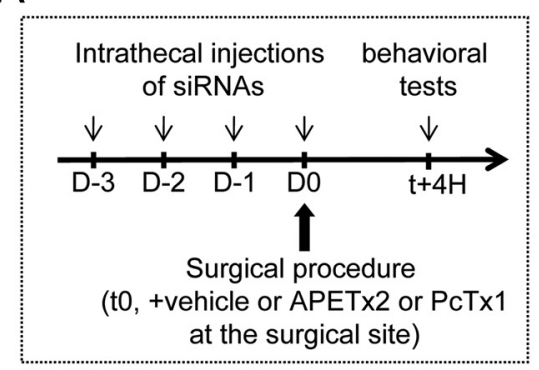

C

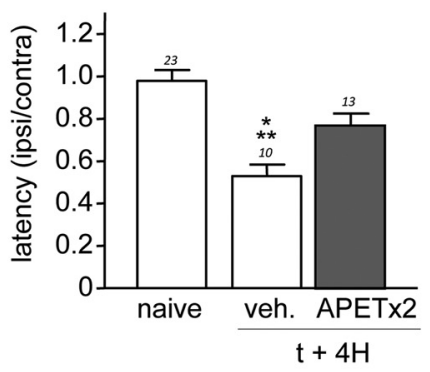

E

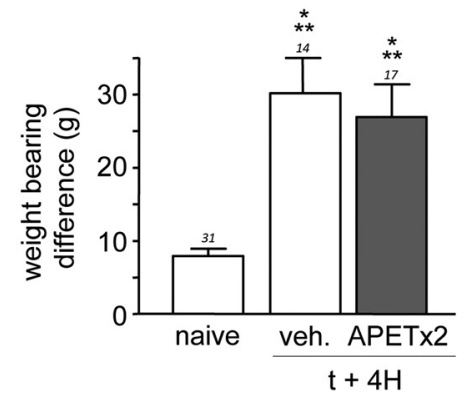

B
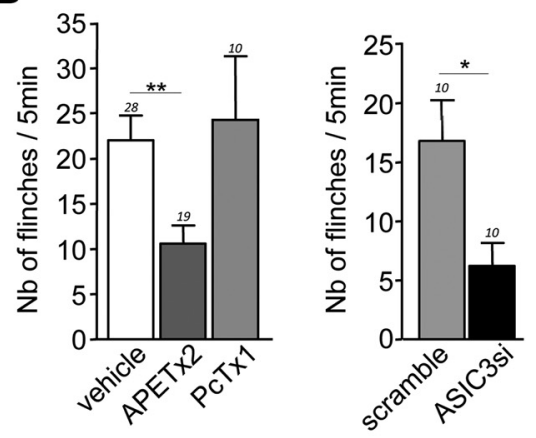

D

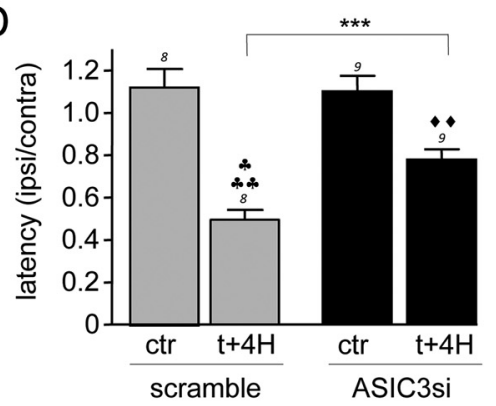

F

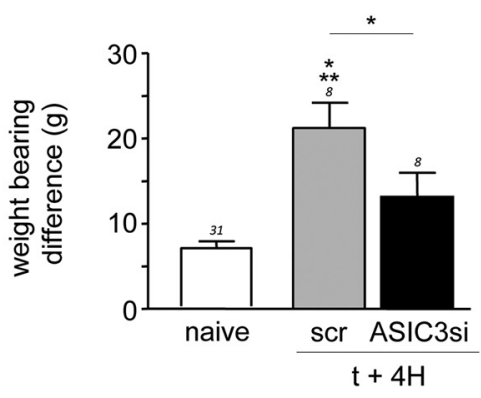

Figure 3. Effect of ASIC3 inhibition on postoperative pain $4 \mathrm{~h}$ after surgery. $\boldsymbol{A}$, Protocol used to test the effect of the ASIC inhibitory toxins (local application during surgery) and of the siRNAs (repetitive intrathecal injections before surgery) on acute postoperative pain ( $4 \mathrm{~h}$ after surgery). B, Effect of APETx2 $20 \mu \mathrm{m}$ and PCTx1 $120 \mathrm{~nm}$ (left), and siRNAs (scramble and ASIC3si; right) on spontaneous pain measured as the number of flinches of the ipsilateral hindpaw over a period of $5 \mathrm{~min}\left({ }^{* *} p<0.01\right.$, KruskalWallis test followed by a Dunn's post hoc test; ${ }^{*} p<0.05$, Mann-Whitney test). C, D, Effect of local application of $20 \mu \mathrm{M}$ APETX2 (C) and ASIC3 knockdown $(\boldsymbol{D})$ on postoperative thermal hyperalgesia evaluated with the Hargreaves test. ( $\left({ }^{* * *} p<0.001\right.$, compared with naive animals, one-way ANOVA followed by a Tukey's post hoc test; $\boldsymbol{D}$ : ${ }^{\star *} p<0.001, \bullet p<0.01$, compared with scramble and ASIC3si controls (ctr), respectively, paired $t$ tests, and ${ }^{* * *} p<0.001$, unpaired $t$ test). $\boldsymbol{E}, \boldsymbol{F}$, Effect of local application of $20 \mu \mathrm{MAPETx} 2(\boldsymbol{E})$ and knockdown of ASIC3 $(\boldsymbol{F})$ on postural pain evaluated with the dynamic weight-bearing test $\left({ }^{*} p<0.05\right.$ and ${ }^{* * *} p<0.001$, one-way ANOVA followed by a Tukey's post hoc test). veh., Vehicle.

previous observation, animals treated with the vehicle displayed primary heat hyperalgesia measured by the Hargreaves test (ratio of ipsilateral to contralateral withdrawal latency significantly reduced in comparison with control animals, $p<0.001$ ) (Fig. $3 C$ ). Intraoperative infusion of APETx 2 induced a modest analgesia since the latency became not significantly different from the one of control animals (Fig. 3C). We also performed a Hargreaves test on animals where ASIC3 expression was knocked down by repetitive intrathecal injections of siRNA (Fig. 3D). The animals that were knocked down still developed heat hyperalgesia after surgery (Fig. $3 D)(p<0.01)$, but the hyperalgesia was significantly attenuated compared with scramble siRNA-treated animals (Fig. $3 D)(p<0.001)$. These data suggest that ASIC3 significantly contributes to primary heat hyperalgesia induced by the surgical procedure, but that inhibition of the channel is not sufficient to fully prevent hyperalgesia.
Surgical procedures provoke postural pain (guarding pain). We have used a weight-bearing test to evaluate postural equilibrium on freely moving rats following surgery (Fig. $3 E, F)$. A difference in weight distribution between the operated and nonoperated paws reflected the intensity of postoperative pain presented by rats. The hindpaw weight-bearing difference measured $4 \mathrm{~h}$ after surgery was abolished in animals treated with ASIC3 siRNA (Fig. $3 F$ ), but was not significantly decreased in animals pretreated with local application of APETx2 (Fig. 3E). This could be explained by a slow onset of the pharmacological inhibition of ASIC3 by intraoperative application of the toxin on the weight-bearing behavior because of delayed toxin availability in deep muscular tissue. Comparatively, the knockdown inhibition obtained with repetitive siRNA treatment was already maximal at the time of surgery. Anyway, the effect of the siRNA suggests a participation of ASIC3 in postoperative postural pain. Together, these data reveal that peripheral inactivation of ASIC3 produces a significant analgesia $4 \mathrm{~h}$ after surgery in a rat model of incisional pain, and therefore support the involvement of ASIC3-containing channels in postoperative pain.

\section{ASIC3 expression is upregulated in the model of postincisional pain}

Animals treated with either local application of vehicle or intrathecal injections of scramble siRNA still experienced pain $24 \mathrm{~h}$ after surgery, showing a hindpaw weight-bearing difference of $\sim 40 \mathrm{~g}(p<$ 0.001 compared with naive animals) (Fig. $4 A$ ). Pain was largely attenuated by both local application of APETx2 during surgery and ASIC3 knockdown (Fig. 4A). We then analyzed modifications in the expression of ASIC3 mRNA and protein in sensory neurons innervating the injured plantar muscle after surgery. RT-qPCR experiments revealed that animals showed an increased level of ASIC3 transcripts in ipsilateral DRGs (L4-L6) compared with their contralateral counterparts $24 \mathrm{~h}$ after surgery (fold expression, ipsilateral vs contralateral DRGs: $1.50 \pm 0.12 ; n=4$ ) (Fig. $4 B$ ), while naive nonoperated animals showed no difference (fold expression: $0.99 \pm 0.12 ; n=5$ ) (Fig. $4 B$ ). Consistent with the increase in ASIC3 transcripts, the number of ASIC3-positive neurons that innervate the incised muscle (i.e., fast blue retrogradely labeled neurons from plantar muscles) was also significantly increased $24 \mathrm{~h}$ after surgery in the ipsilateral L5 DRG (controlateral vs ipsilateral, $74 \pm 2 \%$ and $87 \pm 1 \%$ of fast blue-labeled neurons, respectively; $n=4$ rats; $p<0.05$; at least 268 fast blue-labeled neurons analyzed in each group) (Fig. $4 C$ ). The distribution of ASIC3-expressing neurons innervating the hindpaw plantar muscle was modified in the ipsilateral DRGs $24 \mathrm{~h}$ after surgery with a large increase in the proportion of small-sized neurons 
(Fig. 4D) (118 and 164 neurons analyzed for contralateral and ipsilateral DRGs, respectively). The distribution pattern in contralateral DRGs from operated animals was similar to the pattern in DRGs from naive rats (Fig. 2C). This suggests, together with the similar fraction of fast blue-positive neurons that expressed ASIC3 in naive animals and in contralateral DRG of operated rats $(75 \pm 2 \%$ and $74 \pm 2 \%$, respectively; $n=4$ rats; at least 217 fast blue-labeled neurons analyzed in each group) (Figs. $2 \mathrm{~B}, 4 \mathrm{C}$ ), that the surgery had a unilateral effect on ASIC3 expression, with a selective increase in the DRGs innervating the operated paw. Our observations demonstrate that postoperative pain induced by plantar incision $24 \mathrm{~h}$ after surgery involves peripheral ASIC3 since (1) its expression is significantly increased in sensory neurons innervating the incised muscle, especially in smalldiameter neurons, and since (2) the postoperative pain is abolished when ASIC3 is inactivated.

\section{Discussion}

Postincisional pain models have been very useful to investigate the pathophysiology of postoperative pain (Brennan et al., 1996; Pogatzki et al., 2002; Duarte et al., 2005). One of the best characterized models is the one developed by Brennan et al. (1996), where hindpaw incision induces evoked and nonevoked pain in agreement with clinical evidence (Pogatzki-Zahn et al., 2007). Peripheral and central sensitizations seem to be involved in the development and maintenance of surgical pain (Pogatzki-Zahn et al., 2007). Of particular interest is the local pH decrease at the site of incision (between pH 6.91 and 6.54, depending on the incision model) (Woo et al., 2004), suggesting that $\mathrm{H}^{+}$-gated depolarizing channels in primary afferent neurons such as ASICs and TRPV1 could contribute to postoperative pain.

TRPV1 has been reported to mediate spontaneous activity and heat sensitization of cutaneous primary afferents after plantar incision (Banik and Brennan, 2009), and TRPV1-containing nociceptors have been implicated either in mechanical allodynia and hyperalgesia (Pospisilova and Palecek, 2006) or in heat hyperalgesia and guarding pain (Hamalainen et al., 2009; Kang et al., 2010). The chemosensitivity of C-fibers innervating the rat hindpaw skin also supports a possible implication of ASICs in chemical sensitization (Kang and Brennan, 2009; Kang et al., 2010). This was further suggested by the effect, in the incision model, of amiloride (a poorly selective) and A-317567 (a nondiscriminant) blockers of ASICs (Dubé et al., 2005). We show here that ASIC3 is highly expressed in skeletal muscle afferents of the rat hindpaw, where it is activated by moderate acidifications, and that selective pharmacological block of its activity or in vivo knockdown of its expression produces a significant analgesia after surgery.

ASIC3-containing channels are particularly well suited to detect local changes in $\mathrm{pH}$ associated with postoperative pain and to contribute to nociceptor sensitization: (1) they exhibit the high-
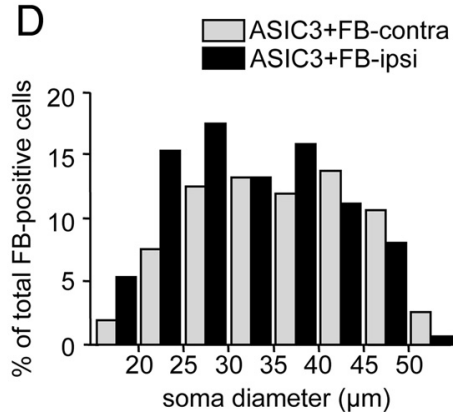
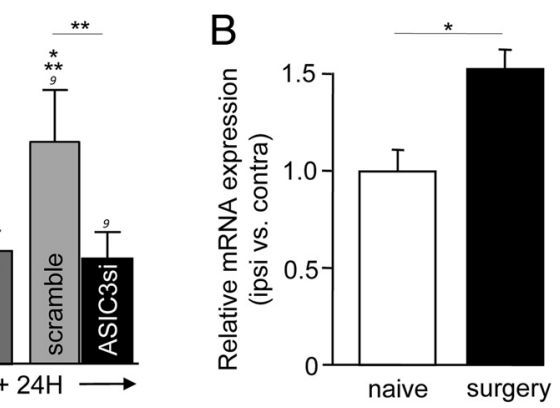
restion

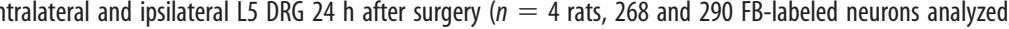
est sensitivity to extracellular protons among ASIC channels (Yagi et al., 2006; Deval et al., 2008; Salinas et al., 2009); (2) they are activated in the range of $\mathrm{pH}$ recorded after skin and/or muscle incision (Woo et al., 2004); and (3) they display a sustained depolarizing current in response to very moderate acidosis that is compatible with the detection of nonadaptating pain. This sustained window current, which is activated for $\mathrm{pH}$ values between 6.5 and 7.4 and is due to the overlap between the pH-dependent activation and inactivation of the channel (Yagi et al., 2006; Deval et al., 2010), has been proposed to contribute to pain during myocardial ischemia and cutaneous inflammation (Yagi et al., 2006; Deval et al., 2008). This window current is blocked by APETx2, as shown in the present study. We have recently shown that peripheral ASIC3 channels in rat skin nociceptors are sensors of acidic pain and are able to integrate several molecular signals produced during inflammation to contribute to primary hyperalgesia (Deval et al., 2008). Although surgical hypersensitivity seems to involve an inflammatory component, the pathophysiological mechanism associated with incision is clearly different from the one causing pain during inflammation (Pogatzki-Zahn et al., 2007). Indeed, inhibition of ASIC3 fully eliminates primary heat hyperalgesia caused by inflammation (Deval et al., 2008), but we report here that it does not completely reverse primary heat hyperalgesia caused by incision. It has also been shown that deep tissue (i.e., muscle) rather than skin incision is critical for the development of guarding pain, which is similar to pain at rest in postoperative patients, and for spontaneous activity in both primary sensory neurons (Xu and Brennan, 2010) and dorsal horn neurons (Xu and Brennan, 2009). Interestingly, ASIC3 has 
been involved in muscular pain caused by repeated acid injections and inflammation in mouse (Sluka et al., 2003, 2007). The more robust effect seen here after knockdown of ASIC3 on weight bearing compared with primary thermal hyperalgesia may thus reflect an important role of ASIC3 in deep tissue, including muscle (Sutherland et al., 2001; Sluka et al., 2003; Ikeuchi et al., 2008), after surgery. However, postural pain that appears $24 \mathrm{~h}$ postsurgery in animals subjected to skin incision alone is also blocked by APETx2 (supplemental Fig. 1, available at www.jneurosci.org as supplemental material), also suggesting a contribution of ASIC3 to prolonged skin pain. ASIC3 thus appears to play an important role in the primary afferent fibers innervating skin and deep tissues such as muscle, sensing pain caused by acidosis and increasing the nociceptive input to the spinal cord, which results in central sensitization. In good agreement with this, experiments performed in rats have reported that activation of nociceptors by acid stimuli induces central sensitization at the level of the dorsal spinal cord (Carpenter et al., 2001), and that deep tissue incision (i.e., plantar muscle) increases both the proportion of dorsal horn neurons with spontaneous activity and the rate of this spontaneous activity (Xu and Brennan, 2009).

Tissue-induced release of mediators, ischemia, and local acidosis are important for primary hypersensitivity and sensitization of nociceptors in the incisional pain models (Woo et al., 2004). A greater response to $\mathrm{pH} 6.0$ and lactic acid of the afferent fibers innervating the rat hindpaw skin has been described after incision (Kang and Brennan, 2009). Lactate enhances the activity of ASIC3 (Immke and McCleskey, 2001), and the increase in tissue lactate concentration (and possibly other mediators) that parallels the decrease in tissue $\mathrm{pH}$ after incision may potentiate the acid-induced activity of ASIC3 (Woo et al., 2004; Kim et al., 2007). This effect can be reinforced by the increased expression of ASIC3 in nociceptors $24 \mathrm{~h}$ after incision that was observed in the present work. Interestingly, nerve growth factor, which is an important regulator of ASIC3 gene expression (Mamet et al., 2002, 2003), has been involved in nociceptor sensitization and heat hyperalgesia after incision (Zahn et al., 2004).

In conclusion, this study involves peripheral ASIC3 channels in postincisional pain. ASIC3 sustained current activated in the $\mathrm{pH}$ range reached after tissue incision is likely to be an important contributor to sensory neuron depolarization and hyperexcitability. Interestingly, a single intraoperative application of an ASIC3 inhibitor evokes a potent analgesic effect $24 \mathrm{~h}$ after surgery. ASIC3 may thus represent a new pharmacological target for the management of postoperative pain in addition to wound infiltration with local anesthetics (Liu et al., 2006; Scott, 2010) and other currently available approaches.

\section{References}

Banik RK, Brennan TJ (2009) Trpv1 mediates spontaneous firing and heat sensitization of cutaneous primary afferents after plantar incision. Pain 141:41-51.

Baron A, Voilley N, Lazdunski M, Lingueglia E (2008) Acid sensing ion channels in dorsal spinal cord neurons. J Neurosci 28:1498-1508.

Bässler EL, Ngo-Anh TJ, Geisler HS, Ruppersberg JP, Gründer S (2001) Molecular and functional characterization of acid-sensing ion channel (ASIC) 1b. J Biol Chem 276:33782-33787.

Bove SE, Flatters SJ, Inglis JJ, Mantyh PW (2009) New advances in musculoskeletal pain. Brain Res Rev 60:187-201.

Brennan TJ, Vandermeulen EP, Gebhart GF (1996) Characterization of a rat model of incisional pain. Pain 64:493-501.

Carpenter KJ, Nandi M, Dickenson AH (2001) Peripheral administration of low $\mathrm{pH}$ solutions causes activation and sensitisation of convergent dorsal horn neurones in the anaesthetised rat. Neurosci Lett 298:179-182.

Chen CC, England S, Akopian AN, Wood JN (1998) A sensory neuron- specific, proton-gated ion channel. Proc Natl Acad Sci U S A 95: $10240-10245$.

Deval E, Noël J, Lay N, Alloui A, Diochot S, Friend V, Jodar M, Lazdunski M, Lingueglia E (2008) ASIC3, a sensor of acidic and primary inflammatory pain. EMBO J 27:3047-3055.

Deval E, Gasull X, Noël J, Salinas M, Baron A, Diochot S, Lingueglia E (2010) Acid-sensing ion channels (ASICs): pharmacology and implication in pain. Pharmacol Ther 128:549-558.

Diochot S, Baron A, Rash LD, Deval E, Escoubas P, Scarzello S, Salinas M, Lazdunski M (2004) A new sea anemone peptide, APETx2, inhibits ASIC3, a major acid-sensitive channel in sensory neurons. EMBO J 23:1516-1525.

Duarte AM, Pospisilova E, Reilly E, Mujenda F, Hamaya Y, Strichartz GR (2005) Reduction of postincisional allodynia by subcutaneous bupivacaine: findings with a new model in the hairy skin of the rat. Anesthesiology 103:113-125.

Dubé GR, Lehto SG, Breese NM, Baker SJ, Wang X, Matulenko MA, Honoré P, Stewart AO, Moreland RB, Brioni JD (2005) Electrophysiological and in vivo characterization of A-317567, a novel blocker of acid sensing ion channels. Pain 117:88-96.

Escoubas P, De Weille JR, Lecoq A, Diochot S, Waldmann R, Champigny G, Moinier D, Ménez A, Lazdunski M (2000) Isolation of a tarantula toxin specific for a class of proton-gated $\mathrm{Na}+$ channels. J Biol Chem 275:25116-25121.

Flatters SJ (2008) Characterization of a model of persistent postoperative pain evoked by skin/muscle incision and retraction (SMIR). Pain 135:119-130.

Hamalainen MM, Subieta A, Arpey C, Brennan TJ (2009) Differential effect of capsaicin treatment on pain-related behaviors after plantar incision. J Pain 10:637-645.

Ikeuchi M, Kolker SJ, Burnes LA, Walder RY, Sluka KA (2008) Role of ASIC3 in the primary and secondary hyperalgesia produced by joint inflammation in mice. Pain 137:662-669.

Ikeuchi M, Kolker SJ, Sluka KA (2009) Acid-sensing ion channel 3 expression in mouse knee joint afferents and effects of carrageenan-induced arthritis. J Pain 10:336-342.

Immke DC, McCleskey EW (2001) Lactate enhances the acid-sensing $\mathrm{Na}+$ channel on ischemia-sensing neurons. Nat Neurosci 4:869-870.

Kang S, Brennan TJ (2009) Chemosensitivity and mechanosensitivity of nociceptors from incised rat hindpaw skin. Anesthesiology 111:155-164.

Kang S, Wu C, Banik RK, Brennan TJ (2010) Effect of capsaicin treatment on nociceptors in rat glabrous skin one day after plantar incision. Pain 148:128-140

Kim TJ, Freml L, Park SS, Brennan TJ (2007) Lactate concentrations in incisions indicate ischemic-like conditions may contribute to postoperative pain. J Pain 8:59-66.

Liu SS, Richman JM, Thirlby RC, Wu CL (2006) Efficacy of continuous wound catheters delivering local anesthetic for postoperative analgesia: a quantitative and qualitative systematic review of randomized controlled trials. J Am Coll Surg 203:914-932.

Mamet J, Baron A, Lazdunski M, Voilley N (2002) Proinflammatory mediators, stimulators of sensory neuron excitability via the expression of acid-sensing ion channels. J Neurosci 22:10662-10670.

Mamet J, Lazdunski M, Voilley N (2003) How nerve growth factor drives physiological and inflammatory expressions of acid-sensing ion channel 3 in sensory neurons. J Biol Chem 278:48907-48913.

Moiniche S, Dahl JB, Erichsen CJ, Jensen LM, Kehlet H (1997) Time course of subjective pain ratings, and wound and leg tenderness after hysterectomy. Acta Anaesthesiol Scand 41:785-789.

Molliver DC, Immke DC, Fierro L, Paré M, Rice FL, McCleskey EW (2005) ASIC3, an acid-sensing ion channel, is expressed in metaboreceptive sensory neurons. Mol Pain 1:35.

Pavlin DJ, Chen C, Penaloza DA, Polissar NL, Buckley FP (2002) Pain as a factor complicating recovery and discharge after ambulatory surgery. Anesth Analg 95:627-634.

Perkins FM, Kehlet H (2000) Chronic pain as an outcome of surgery. A review of predictive factors. Anesthesiology 93:1123-1133.

Pogatzki EM, Niemeier JS, Brennan TJ (2002) Persistent secondary hyperalgesia after gastrocnemius incision in the rat. Eur J Pain 6:295-305.

Pogatzki-Zahn EM, Zahn PK, Brennan TJ (2007) Postoperative painclinical implications of basic research. Best Pract Res Clin Anaesthesiol 21:3-13. 
Pospisilova E, Palecek J (2006) Post-operative pain behavior in rats is reduced after single high-concentration capsaicin application. Pain 125:233-243.

Salinas M, Lazdunski M, Lingueglia E (2009) Structural elements for the generation of sustained currents by the acid pain sensor ASIC3. J Biol Chem 284:31851-31859.

Scott NB (2010) Wound infiltration for surgery. Anaesthesia 65 [Suppl $1]: 67-75$.

Sluka KA, Price MP, Breese NM, Stucky CL, Wemmie JA, Welsh MJ (2003) Chronic hyperalgesia induced by repeated acid injections in muscle is abolished by the loss of ASIC3, but not ASIC1. Pain 106:229-239.

Sluka KA, Radhakrishnan R, Benson CJ, Eshcol JO, Price MP, Babinski K, Audette KM, Yeomans DC, Wilson SP (2007) ASIC3 in muscle mediates mechanical, but not heat, hyperalgesia associated with muscle inflammation. Pain 129:102-112.

Sutherland SP, Benson CJ, Adelman JP, McCleskey EW (2001) Acidsensing ion channel 3 matches the acid-gated current in cardiac ischemiasensing neurons. Proc Natl Acad Sci U S A 98:711-716.

Voilley N, de Weille J, Mamet J, Lazdunski M (2001) Nonsteroid antiinflammatory drugs inhibit both the activity and the inflammationinduced expression of acid-sensing ion channels in nociceptors. J Neurosci 21:8026-8033.

Waldmann R, Champigny G, Bassilana F, Heurteaux C, Lazdunski M (1997a) A proton-gated cation channel involved in acid-sensing. Nature 386:173-177.

Waldmann R, Bassilana F, de Weille J, Champigny G, Heurteaux C, Lazdunski M (1997b) Molecular cloning of a non-inactivating proton-gated $\mathrm{Na}+$ channel specific for sensory neurons. J Biol Chem 272:20975-20978.

Woo YC, Park SS, Subieta AR, Brennan TJ (2004) Changes in tissue pH and temperature after incision indicate acidosis may contribute to postoperative pain. Anesthesiology 101:468-475.

Wu CL, Naqibuddin M, Rowlingson AJ, Lietman SA, Jermyn RM, Fleisher LA (2003) The effect of pain on health-related quality of life in the immediate postoperative period. Anesth Analg 97:1078-1085.

Xu J, Brennan TJ (2009) Comparison of skin incision vs. skin plus deep tissue incision on ongoing pain and spontaneous activity in dorsal horn neurons. Pain 144:329-339.

Xu J, Brennan TJ (2010) Guarding pain and spontaneous activity of nociceptors after skin versus skin plus deep tissue incision. Anesthesiology 112:153-164.

Yagi J, Wenk HN, Naves LA, McCleskey EW (2006) Sustained currents through ASIC3 ion channels at the modest $\mathrm{pH}$ changes that occur during myocardial ischemia. Circ Res 99:501-509.

Zahn PK, Subieta A, Park SS, Brennan TJ (2004) Effect of blockade of nerve growth factor and tumor necrosis factor on pain behaviors after plantar incision. J Pain 5:157-163. 